\title{
DEPLOYMENT AND PERFORMANCE OF THE NASA D3R DURING GPM IFLOODS FIELD CAMPAIGN
}

\author{
V. Chandrasekar ${ }^{1}$, Haonan Chen ${ }^{1}$, Robert M. Beauchamp ${ }^{1}$, Manuel Vega $^{1,2}$, \\ Mathew Schwaller ${ }^{2}$, Walter Petersen ${ }^{3}$, David Wolff $^{3}$, Delbert Willie ${ }^{1}$ \\ ${ }^{1}$ Colorado State University, Fort Collins, CO \\ ${ }^{2}$ NASA Goddard Space Flight Center, Greenbelt, MD \\ ${ }^{3}$ NASA Wallops Flight Facility, Wallops Island, VA
}

\begin{abstract}
The Iowa Flood Studies (IFloodS) field experiment was conducted to better understand the strengths and limitations of Global Precipitation Measurement (GPM) mission satellite products in the context of hydrologic applications. The NASA dual-frequency dual-polarization Doppler radar (D3R), designed as part of the GPM ground validation program, participated in the IFloodS field campaign to characterize precipitation properties at $\mathrm{Ku}$ - and Ka-band frequencies. This paper presents the deployment of the D3R and summarizes the D3R observations during the IFloodS field campaign. The quality of the D3R measurements is evaluated by comparing with the NASA NPOL S-band radar observations. In addition, the capability for rainfall estimation using the $\mathrm{D} 3 \mathrm{R}$ is also described and validated using ground gauge measurements.
\end{abstract}

Index Terms - Radar, D3R, GPM, ground validation, IFloodS, rainfall, multi-frequency

\section{INTRODUCTION}

The NASA dual-frequency dual-polarization Doppler radar (D3R) [1] is a ground validation radar designed to support the ground validation (GV) activities of NASA's Global Precipitation Measurement (GPM) mission. The D3R is a fully polarimetric, scanning weather radar system operating at the nominal frequencies of $13.91 \mathrm{GHz}$ and $35.56 \mathrm{GHz}$ covering a maximum range of about $40 \mathrm{~km}$. The $\mathrm{D} 3 \mathrm{R}$ frequencies were chosen to match the GPM dual-frequency precipitation radar (DPR) system which was launched as an instrument on the core observatory in February, 2014. The D3R takes advantage of several innovative technologies to achieve its design goals as a relatively compact, transportable system. Chief among these innovations are the use of solid state power amplifiers and a novel waveform composed of three consecutive, frequency modulated, frequency separated pulses. Using these methods, blind ranges and range side lobes are minimized, and the radar meets its sensitivity requirement of $-10 \mathrm{dBZ}$

This work is sponsored by the NASA GPM project. at $15 \mathrm{~km}$ (clear air, single pulse, with $150 \mathrm{~m}$ range resolution) [3]. The D3R ground-based radar is expected to provide detailed, fine-scale statistics of precipitation while the GPM DPR presents a global picture of precipitations through the $\mathrm{Ku}$ - and Ka-band observations.

As part of the GV program for the GPM project, the Iowa Flood Studies (IFloodS) field campaign took place in eastern Iowa from May 1, 2013, through June 15, 2013. This was a multi-organization field experiment conducted by NASA, coordinated by the Iowa Flood Center. In IFloodS, a vast network of ground instruments were deployed. These included multiple radars, rain gauges, and disdrometers, with a focus on measuring surface precipitation. The radars and ground instruments were situated to monitor precipitation within the Cedar and Iowa River basins between Waterloo, Iowa, and Iowa City, Iowa. Additional ground instruments were placed throughout the Turkey River basin in northeast Iowa. This comprehensive data set is being used with flood and forecasting models to better understand the capabilities and limitation for flood forecasting.

During the IFloodS field campaign, the D3R radar was colocated with the NASA polarimetric (NPOL) S-band radar to enable triple-frequency evaluation of precipitation along with a array of rain gauges and disdrometers to measure precipitation at the ground. In addition, the radar measurements provide a more detailed understanding of the microphysical properties of precipitation and will be used to refine and improve the algorithms used on the GPM core observatory satellite. A description about the potential of dual-polarization radar in the validation of satellite precipitation measurements can be found in [2].

This paper presents the observations of the D3R radar during the IFloodS experiment. Specifically, the accuracy of D3R measurements such as reflectivity, differential reflectivity, and differential propagation phase is presented, with particular attention paid comparing D3R and NPOL observations. Preliminary rainfall estimates and validations against ground in-situ samples are also described to demonstrate the capability of the D3R for rainfall measurement. 


\section{D3R OBSERVATIONS DURING IFLOODS}

Figure 1 shows the NASA D3R and NPOL radar pictures as well as the locations of the radars and a subset of ground insitu sampling devices during the IFloodS field experiment. During this campaign, the D3R performed more than 45,000 scans covering an azimuth range of 90 to 230 degrees and collected over 18 TB of data. Precipitation was observed and collected on 35 days of the 46 day experiment. Data collection was performed in coordination with the NPOL radar. The precipitation data includes a minimum of five severe convective thunderstorms, two squall lines, and four widespread stratiform rain events.
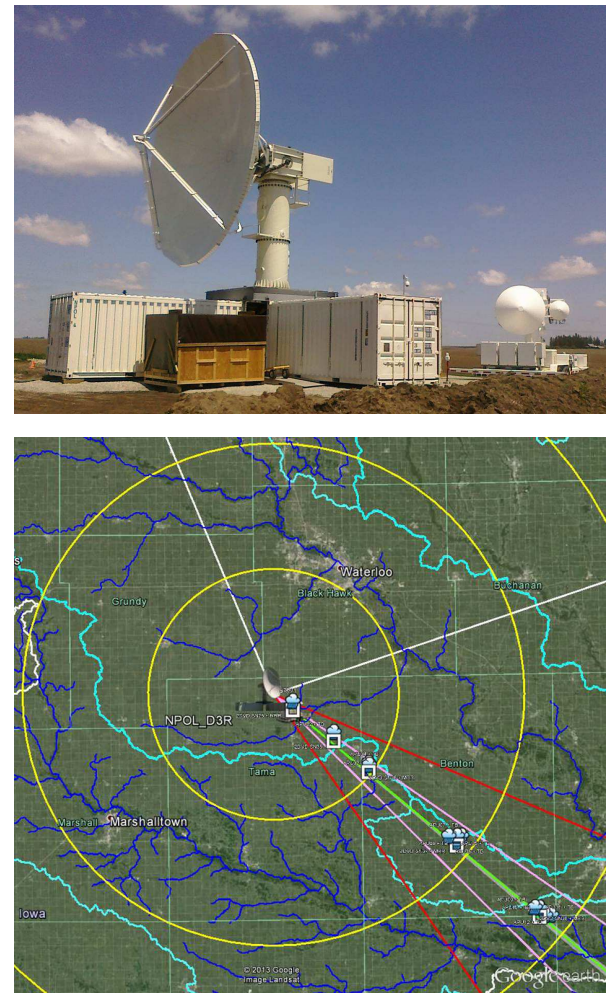

Fig. 1. D3R and NPOL co-located in Traer, IA, during the IFloodS field campaign. $25 \mathrm{~km}$-range rings in yellow with the D3R and NPOL radars located at the center.

\section{ATTENUATION CORRECTION AND MULTI-FREQUENCY COMPARISONS}

In recent years, radar systems operating at higher frequencies such as $\mathrm{X}$ - or $\mathrm{Ku}$-band are gaining an increasing interest in the weather radar community because of the cost efficiency and compact configurations. However, the signal attenuation in heavy rainfall needs to be compensated for. The radar observations should be corrected for attenuation prior to using them for rainfall estimation.
In this paper, we use the self-consistency technique to check the quality of radar measured dual-polarization variables. The self-consistency technique is based on the concept that the dual-polarization measurements of $Z, Z_{d r}$, and $K_{d p}$ lie in a limited three-dimensional space for rain medium [4]. In addition, a differential phase based attenuation correction algorithm has been developed for the D3R radar. Figure 2(a) shows the scatter density plot of attenuation corrected $Z_{d r}$ versus attenuation corrected $Z$ for a sample plan position indicator (PPI) scan from the D3R Ku-band at 02:59UTC, May 20, 2013. Figure 2(b) is the scatter density plots of $K_{d p}$ versus $Z$ after attenuation correction for the same data. The black dots shown in Figures 2(a) and (b) are simulated radar moments from in-situ ground instrument DSD observations. The consistency between the real radar observations and the simulated radar moments using DSD data provides validation that the radar data after attenuation correction is of high quality.

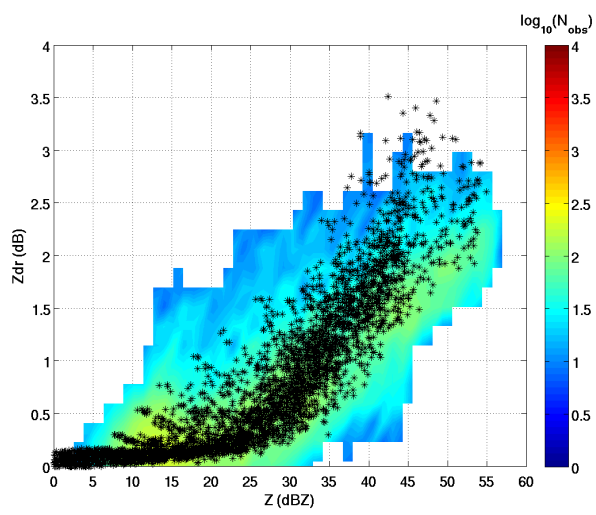

(a) Attenuation Corrected $Z_{d r}$ versus Attenuation Corrected $Z$

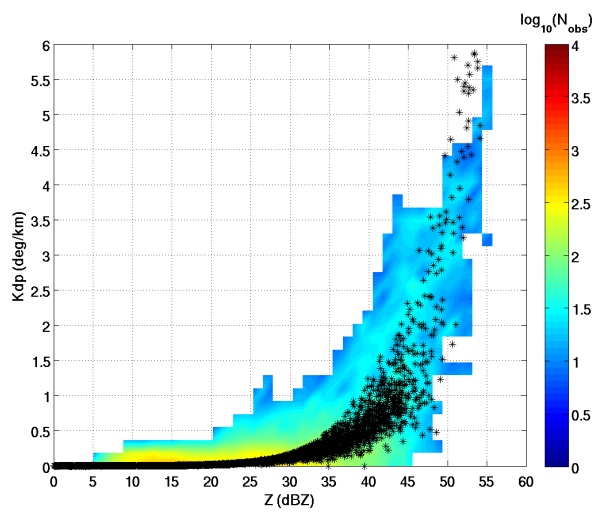

(b) $K_{d p}$ versus Attenuation Corrected $Z$

Fig. 2. Scatter density plots of (a) attenuation corrected $Z_{d r}$ versus attenuation corrected $Z$ (b) $K_{d p}$ versus attenuation corrected $Z$ for D3R Ku-band radar measurements at 02:59UTC, May 20, 2013. The black dots are simulated radar moments from the APU DSD observations.

In addition, a multi-frequency comparison has been con- 
ducted to further demonstrate the accuracy of D3R measurements. Figure 3 shows a sample range height indicator (RHI) plots of reflectivity for NPOL and D3R radar observations at 13:25UTC, May 03, 2013. Figure 4 illustrates a sample PPI observation from NPOL and D3R radar at 22:48UTC, May 29, 2013. Figure 5 shows a sample comparison of observed reflectivity profiles between NPOL and the D3R along a single ray from the PPI shown in Figure 4. From the sample ray plot, we can conclude that the D3R measurements match the NPOL observations very well.

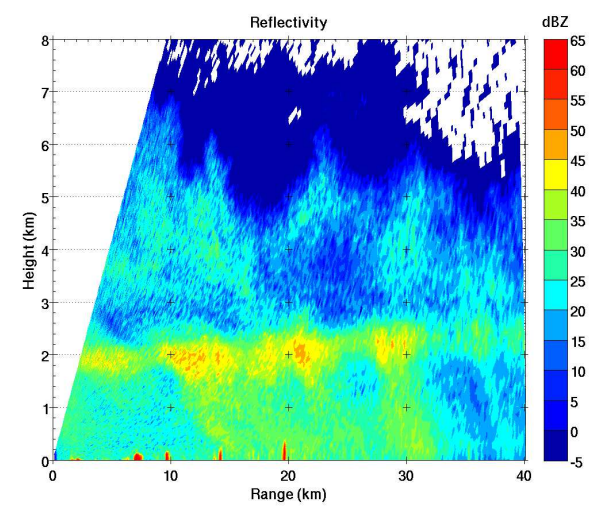

(a) NPOL Observation

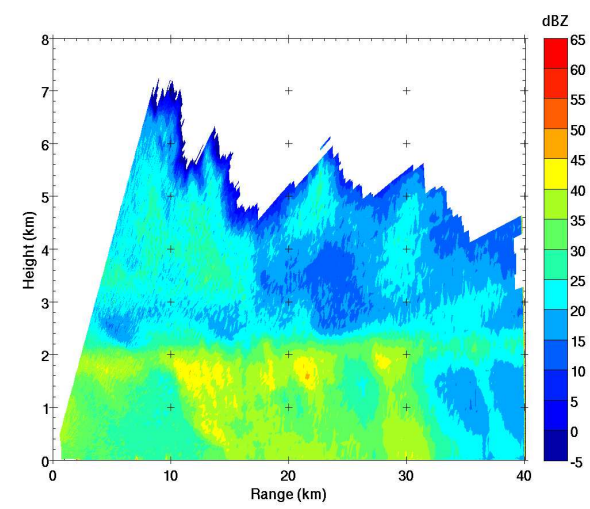

(b) D3R Observation

Fig. 3. (a) NPOL observation (b) D3R Ku-band Observation at 13:25UTC, May 03, 2013.

\section{RAINFALL ESTIMATES AND VALIDATIONS}

In order to derive the rainfall retrieval algorithm for D3R, sample rain drop size distribution (DSD) data from an autonomous parsivel unit (APU) collected during the IFloodS field campaign is used for illustration purposes. In this study, a total of 11388 one-minute-averaged DSDs are used to simulate the dual-polarization radar moments and compute theoretical rainfall rate values. This DSD data set includes 8 precipitation events/days during the IFloodS field experiment.

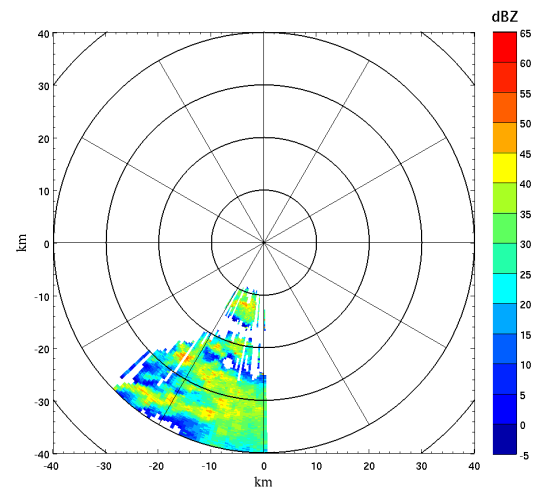

(a) NPOL Observation

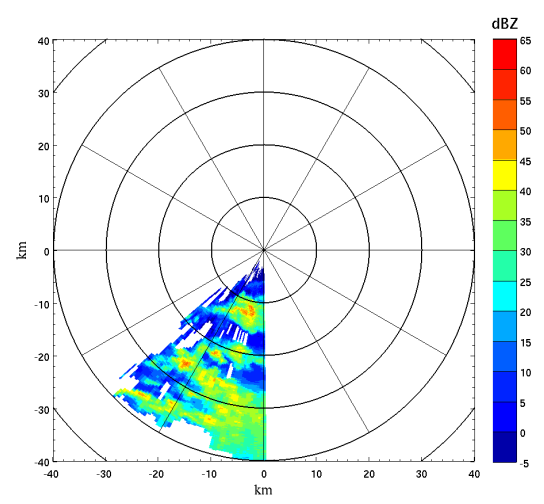

(b) D3R Observation

Fig. 4. (a) NPOL observation (b) D3R Ku-band Observation at 22:48UTC, May 29, 2013.

Figure 6 shows a scatter density plot of rainfall rate versus specific differential phase at Ku-band based on the DSD observations. The best-fit $R\left(K_{d p}\right)$ relation is in the power-law form:

$$
R=10.25 K_{d p}^{0.90}
$$

In this paper, the storm event that occurred on May 29, 2013 is analyzed as an example demonstrating the capability of D3R for measuring rainfall. For validation purposes, the 5-min rainfall amounts observed by a tipping-bucket rain gauge is used for cross comparisons between the D3R rainfall products and the gauge records. In order to quantify the error of radar rainfall products, we use the normalized standard error of rainfall (NSE) to evaluate the radar rainfall performance. Assuming the rain gauge measurements are the truth, the NSE can be defined in the form:

$$
N S E=\frac{\frac{1}{N} \sum\left|R_{n}-G_{n}\right|}{\frac{1}{N} \sum G_{n}}
$$

where $R_{n}$ and $G_{n}$ denote the radar estimate and gauge measurement of rainfall at time frame $n$, respectively. $N$ is the 


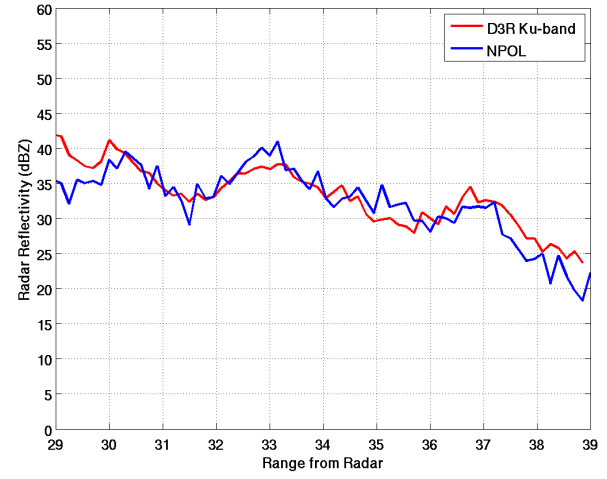

Fig. 5. Cross comparison between NPOL and D3R Kuband reflectivity measurement (azimuth $=190$ degree) at 22:48UTC, May 29, 2013

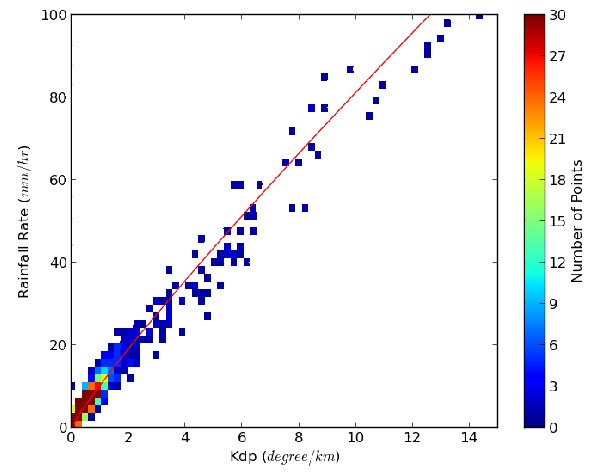

Fig. 6. Scattergram of rainfall rate versus $K_{d p}$ based on the simulation data from DSD observations

total sample number of radar and gauge rainfall estimates.

For Ku-band rainfall product, the $N S E$ is computed based on data collected over the May 29, 2013 rainfall event. The 5-min rainfall products has an NSE of about $15.7 \%$, and the accumulation of 7 measurements of 5-min rainfall (35-min rainfall accumulation) has an NSE of about $2 \%$.

\section{SUMMARY}

The GPM core observatory was launched in February 27, 2014. The goal of the GPM mission is to provide accurate and frequent observations of global precipitation which will play an important role in improving weather, climate, and hydrological prediction capabilities. As an indispensable part of the GPM project, ground validation will focus on the demonstration and evaluation of space based precipitation estimation and classification algorithms. From May 1 to June 15, 2013, a multi-organizational field experiment, coordinated by NASA and the Iowa Flood Center, was deployed to collect precipitation data at the Earth's surface using ground instruments, advanced weather radars, and satellites passing overhead.

This paper described the deployment and observations of NASA D3R during the IFloodS field campaign. The D3R is an advanced dual-polarization weather radar system operating at $\mathrm{Ku}$ - and $\mathrm{Ka}$-band frequencies, which has great potential for understanding the precipitation microphysics and as a cross validation platform for comparison with space based observations. Cross comparison between the D3R observations and NPOL S-band radar observations demonstrated the measurement accuracy of the D3R. In addition, an $R\left(K_{d p}\right)$-based rainfall algorithm has been developed for D3R using the DSD data collected by ground disdrometers. The QPE evaluation results based on the gauge observations show excellent performance of the rainfall products from the D3R with low normalized standard error.

\section{REFERENCES}

[1] V. Chandrasekar, Mathew Schwaller, Manuel Vega, James Carswell, Kumar Vijay Mishra, Robert Meneghini, and Cuong Nguyen, 2011, "Scientific and engineering overview of the NASA dual-frequency dualpolarized doppler radar (D3R) system for GPM ground validation," IEEE International Geoscience and Remote Sensing Symposium, Vancouver, Canada, July 24-29.

[2] V. Chandrasekar, V. N. Bringi, S. A. Rutledge, Arthur Hou, Eric Smith, Gail Skofronick Jackson, E. Gorgucci, and W. A. Petersen, 2008, "Potential Role Of Dual- Polarization Radar In The Validation Of Satellite Precipitation Measurements: Rationale and Opportunities,"Bull. Amer. Meteor. Soc., 89, 1127-1145.

[3] N. Bharadwaj and V. Chandrasekar, 2007, "Phase Coding for Range Ambiguity Mitigation in Dual-Polarized Doppler Weather Radars,"J. Atmos. Oceanic Technol., 24, 1351-1363.

[4] G. Scarchilli, E. Gorgucci, and V. Chandrasekar, 1996, "Self-consistency of polarization diversity measurement of rainfall,"IEEE Trans. Geosci. Remote Sens., 30, 2226. 